\title{
Lipiodol nanoemulsions stabilized with polyglycerol-polycaprolactone block copolymers for theranostic applications
}

Trang Huyen Le Kim ${ }^{1}$, Hwiseok Jun ${ }^{1}$, Jin Ho Kim², Keunchil Park ${ }^{3}$, Jee Seon Kim ${ }^{1 *}$ (D) and Yoon Sung Nam ${ }^{4^{*}}$

\begin{abstract}
Background: Polyglycerol is an attractive hydrophilic building block of amphiphilic copolymers for biomedical and pharmaceutical applications due to its biocompatibility, facile chemical modification, and anti-fouling activity. Herein we introduce theranostic nanoemulsions incorporating anti-cancer therapeutic and contrast agents using linear polyglycerol-poly( $\varepsilon$-caprolactone) diblock copolymers (PG-b-PCL). Lipiodol is used as a core oil that dissolves paclitaxel and serves as a contrast agent for computer tomography (CT).

Methods: PG-b-PCL is synthesized by three-step processes: polymerization of ethoxyethyl glycerol ether; ringopening polymerization of $\varepsilon$-caprolactone; and deprotection of the PEEGE block. In vitro cytotoxicity of the polyglycerolated lipiodol nanoemulsions is demonstrated using HeLa ovarian cancer cells. The applicability of the prepared nanoemulsions as a contrast agent for CT imaging is also evaluated using micro-CT.

Results: Three compositions of PG-b-PCL with different block lengths are synthesized to prepare nanoemulsions. The polyglycerolated lipiodol nanoemulsions exhibit excellent anti-cancer activities, while placebo nanoemulsions have no significant cytotoxicity under the same condition. Micro-CT imaging of the nanoemulsions confirms the ability of nanoemulsions as a contrast agent.

Conclusions: This study suggests that PG-b-PCL is a promising polymeric emulsifier for effective stabilization and surface functionalization of drug delivery nanocarriers for therapeutic and imaging agents.
\end{abstract}

Keywords: Polyglycerol, Amphiphilic copolymers, Nanoemulsions, Lipiodol, Paclitaxel, Cancer

\section{Background}

Recently, various amphiphilic copolymers have been studied for drug delivery with aiming to enhancing the therapeutic effects of drugs through long-term blood circulation and facile surface modification [1-3]. Polyethylene glycol (PEG) has been extensively employed as the hydrophilic, non-sticky corona of drug delivery nanocarriers because PEG efficiently suppresses non-specific protein adsorption and colloidal aggregation in the blood stream [4]. However, recent studies showed that a long PEG chain can effectively

\footnotetext{
* Correspondence: eliekim@kaist.ac.kr; yoonsung@kaist.ac.kr

'Department of Materials Science and Engineering, Korea Advanced Institute of Science and Technology, 291 Daehak-ro, Yuseong-gu, Daejeon 34141, Republic of Korea

${ }^{4}$ Department of Materials Science and Engineering and KAIST Institute for the NanoCentury, Korea Advanced Institute of Science and Technology, 291 Daehak-ro, Yuseong-gu, Daejeon 34141, Republic of Korea

Full list of author information is available at the end of the article
}

hinder the cellular uptake of nanoparticles and the following endosomal escape partially owing to its neutrality or lack of responsiveness to environmental changes [5-7]. In addition, although cell adhesion molecules are generally introduced at the end of PEG exposed to the surface, the number of molecules that can be conjugated is very limited, and relatively large, hydrophobic targeting molecules can negatively affect the self-assembly and surface properties of nanocarriers [8-10]. Therefore, alternative hydrophilic polymers have been explored to overcome the limitations of PEG.

Polyglycerol (PG) has received increasing attention as a new hydrophilic polymer due to its unique chemical properties [11-14]. These include a large number of hydroxyl groups, dendritic structures [15], and biocompatibility [16]. PG was also co-polymerized with poly( $\varepsilon$ caprolactone) (PCL) [17], PCL-polycitric [18], and PEG 
[19] to enhance the intracellular delivery of anti-cancer drugs [20]. In comparison to PEG, the hydroxyl groups on each monomeric unit of PG can help increase the hydrophilic properties of the nanoparticle surface, thereby increasing the dispersion stability of nanocarriers in the presence of serum proteins, and provide multiple sites for the conjugation of targeting molecules.

In this work, we employed a linear PG-PCL diblock copolymer (PG- $b$-PCL) as an emulsifier to stabilize lipiodol nanoemulsions and functionalize the PG block with cancer cell-targeting molecules. Lipiodol, an iodinated derivative of poppy seed oil with a high iodine content (30-40 wt-\%) [21], has been used as a clinically approved computer tomography $(\mathrm{CT})$ contrast-enhancing agent and has been used for trans-arterial chemoembolization for hepatocellular carcinoma [22]. Recently, theranostic nanocarriers have been vigorously investigated for the co-delivery of imaging and therapeutic agents to tumor tissues, enabling to identify the precise location of tumors in a non-invasive manner and provide evidences to determine a suitable treatment regimen [23]. Our previous studies demonstrated that PEG-PCL diblock copolymers can form a semi-solid interphase between oil and water, producing robust oil-in-water emulsions [24-27]. The present work extended the same concept of emulsion stabilization to PG- $b$-PCL with lipiodol as a core oil of the nanoemulsions dissolving paclitaxel [28-30]. The cytotoxicity and cellular uptake of the prepared nanoemulsions were investigated to demonstrate the feasibility of lipiodol nanoemulsions stabilized with PG- $b$-PCL as a delivery carrier for anti-cancer drugs.

\section{Methods}

\section{Materials}

Ethyl vinyl ether (99\%), glycidol (96\%), $p$-toluene sulfonic acid monohydrate ( $\mathrm{TsOH}, 98 \%)$, oxalic acid $(98 \%)$, potassium tert-butoxide ( $>98 \%), \varepsilon$-caprolactone (97\%), tin(II) 2 ethylhexanoate (92.5-100\%), 3-aminophenylboronic acid hydrochloride (3-APBA, 98\%), folic acid (97\%), $\mathrm{N}$-(3dimethylaminopropyl)- $N$ '-ethylcarbodiimidehydrochloride (EDC, 98\%), $N$-hydroxysuccinimide (NHS), and 4',6diamidino-2-phenylindole (DAPI), were purchased from Sigma-Aldrich (St. Louis, Mo, USA). Lipiodol was purchased from Guerbet (Aulnay-sous-Bois, France), and paclitaxel (>97\%) from Wako Pure Chemical Industries, Ltd. (Osaka, Japan). Human cervical epithelial carcinoma (HeLa) cells were purchased from the Korean Cell Line Bank (Seoul, Republic of Korea). A Cell-Counting Kit-8 (CCK-8) was obtained from Dojindo Laboratories (Kumamoto, Japan). Dulbecco's modified Eagle's medium (DMEM), phosphate buffered saline (PBS), and fetal bovine serum (FBS) were purchased from Invitrogen (Carlsbad, CA, USA).

\section{Synthesis of linear PG-b-PCL}

Ethoxyethyl glycerol ether (EEGE) was synthesized from glycidol and ethyl vinyl ether using $\mathrm{TsOH}$ as a catalyst at room temperature for $3 \mathrm{~h}$. The product was washed with saturated $\mathrm{NaHCO}_{3}$ and then isolated using ethyl acetate and dried using $\mathrm{MgSO}_{4}$, followed by distillation at $64{ }^{\circ} \mathrm{C}$ in vacuum. Poly(ethoxyethyl glycerol ether) (PEEGE) was synthesized through polymerization of EEGE in THF at $65^{\circ} \mathrm{C}$ for $24 \mathrm{~h}$ using potassium tert-butoxide as an initiator. The produce was precipitated in cold methanol, and then the solvent was evaporated at $30{ }^{\circ} \mathrm{C}$. The synthesized PEEGE was isolated using benzene and dried in vacuum for $24 \mathrm{~h}$. Linear PEEGE- $b$-PCL was synthesized by the ring opening polymerization of $\varepsilon$-caprolactone at $125{ }^{\circ} \mathrm{C}$ for $20 \mathrm{~h}$ using PEEGE and stannous octanoate as an initiator and a catalyst, respectively. The synthesized polymer was dissolved in methylene chloride and precipitated in cold hexane. The de-protection of PEEGE- $b$-PCL was carried out to obtain PG- $b$-PCL by mixing the PEEGE- $b$-PCL solution in acetone with an aqueous solution of oxalic acid. The mixture solution was magnetically stirred for $5 \mathrm{~h}$ at room temperature. The mixture was then dialyzed against deionized water through a Spectra/Por membrane for $24 \mathrm{~h}$. The final polymer product was dried by rotary evaporation.

\section{Characterization of PG- $b$-PCL}

Gel permeation chromatography (GPC) analysis was performed using Agilent high performance liquid chromatography (HPLC) 110 series (Agilent Technologies, Palo Alto, CA, USA). Three PL gel columns $(300 \times 7.5 \mathrm{~mm}$, pore sizes $=10^{3}, 10^{4}$, and $10^{5} \AA$ ) and a refractive index detector were used. Tetrahydrofuran was used as an isocratic

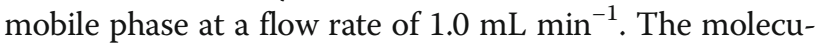
lar weight of the polymers was obtained through calibration against monodispersed polystyrene standards (Polysciences, Inc., Warrington, PA, USA). ${ }^{1} \mathrm{H}$ Nuclear magnetic resonance (NMR) analysis was performed at $25{ }^{\circ} \mathrm{C}$ using a Bruker NMR (300 MHz) using $\mathrm{CDCl}_{3}$ as a solvent. Chemical shifts were measured in parts per million (ppm) using tetramethylsilane as an internal reference. Differential scanning calorimetry (DSC) analysis was performed using DSC 204 F1 Phoenix (Netzsch- Gerätebau GmbH, Selb, Germany). Heating and cooling scans were run between -50 and $200{ }^{\circ} \mathrm{C}$ at a scan rate of $10{ }^{\circ} \mathrm{C} \mathrm{min}^{-1}$.

\section{Preparation of Lipiodol Nanoemulsions}

Various preparation conditions for placebo nanoemulsions (denoted as 'bNEs') and paclitaxel-incorporating nanoemulsions (denoted as 'pNEs') were investigated. Briefly, the pre-determined amounts of PG- $b$-PCL, lipio$\mathrm{dol}$, and paclitaxel were dissolved completely in acetone by sonication for $10 \mathrm{~min}$. The solutions were then added dropwisely into $10 \mathrm{~mL}$ deionized water with vigorous 
magnetic stirring or homogenization. Various stirring/ homogenizing speeds (500-10,000 rpm) and temperatures $\left(25-50{ }^{\circ} \mathrm{C}\right)$ were examined. The mixtures were then stirred overnight for solvent evaporation. The produced nanoemulsions were stored at $4{ }^{\circ} \mathrm{C}$ until use.

\section{Characterization of Lipiodol Nanoemulsions}

The hydrodynamic size distribution of the nanoemulsions was determined by dynamic laser light scattering (DLS) using a particle size analyzer ELSZ-1000 (Otsuka Electronics Co., Ltd., Osaka, Japan). The morphology of the nanonemulsions was observed via transmission electron microscopy (TEM) (JEM-3010, JEOL, Japan). TEM specimen was prepared by mixing $10 \mu \mathrm{L}$ of the nanoemulsions with $10 \mu \mathrm{L}$ of $2 \%$ uranyl acetate for negative staining. The mixture was dropped on a carbon-coated copper grid (Ted Pella, Inc., Redding, CA, USA), followed by incubation at room temperature for $5 \mathrm{~min}$. The drop was removed using a filter paper, and the grids were dried in air.

\section{Determination of loading yield and efficiency}

The nanoemulsions $(500 \mu \mathrm{L})$ were freeze-dried and dissolved in $1.5 \mathrm{~mL}$ ethanol with sonication for $1 \mathrm{~h}$. The solution was filtered through a $0.45 \mu \mathrm{m}$ syringe filter, and the amount of lipiodol was determined at $260 \mathrm{~nm}$ using UV-visible absorption spectrophotometry (UV-1601, Shimadzu, Kyoto, Japan). As for the loading yield and efficiency of paclitaxel, the nanoemulsions $(1 \mathrm{~mL})$ were freeze-dried and dissolved in $1 \mathrm{~mL}$ acetonitrile with sonication for $1 \mathrm{~h}$. The solution was filtered through a $0.45 \mu \mathrm{m}$ syringe filter, paclitaxel in the filtrate was determined using reversed phase HPLC (Agilent 1100 series, Agilent Technologies, Palo Alto, USA) equipped with a Waters Spherisorb ODS2 column $(\mathrm{C} 18,5 \mu \mathrm{m}$, $4.6 \mathrm{~mm} \times 250 \mathrm{~mm}$ ). Acetonitrile was used as an isocratic mobile phase at a rate of $1 \mathrm{~mL} \mathrm{~min}{ }^{-1}$. The absorption of the eluted solution was determined at $227 \mathrm{~nm}$ using a standard curve of paclitaxel in acetonitrile.

\section{Conjugation of folic acids to Nanoemulsions}

Folic acid (FA, $1 \mathrm{mg}$ ) was dissolved in $N, N$-dimethylformamide (DMF, $5 \mathrm{~mL}$ ) at room temperature with magnetic stirring for $20 \mathrm{~min}$. EDC (440 mg) and NHS $(260 \mathrm{mg}$ ) was added to the solution, and then the solution was magnetically stirred on ice for $1 \mathrm{~h}$. Then, 3 APBA (411 mg) in DMF (2 mL) was then mixed with the FA solution followed by stirring in an ice bath for $1 \mathrm{~h}$ and at room temperature for $24 \mathrm{~h}$. The solution was then added dropwisely into deionized water $(50 \mathrm{~mL})$. Yellow precipitates were gathered by filtration and then washed several times with acetone. The folate boronic acid (FBA) powder was dried under vacuum overnight, dissolved in DMSO, and then diluted by deionized water to the final concentration of $20 \mu \mathrm{g} \mathrm{mL} \mathrm{L}^{-1}$. The FBA solution $(5 \mathrm{~mL})$ was mixed with pNEs $(1 \mathrm{~mL})$ by magnetic stirring at room temperature for $24 \mathrm{~h}$ for esterification, and then non-conjugated FBA were removed by dialysis against deionized water through a Spectra/Por membrane $(\mathrm{MWCO}=12 \mathrm{kDa})$ for $12 \mathrm{~h}$. To determine the degree of FA substitution, the prepared FA-conjugated pNEs (denoted as 'fpNEs') were freeze-dried and then re-dissolved in DMSO by sonication for $15 \mathrm{~min}$. The UV absorbance of folates was measured at $363 \mathrm{~nm}$, and the number of FA conjugated to the nanoemulsions was calculated using a calibration curve of FA in DMSO.

\section{Serum stability and in vitro release}

The serum stability of pNEs was determined by incubating the nanoemulsions in PBS containing 10\% FBS at $37{ }^{\circ} \mathrm{C}$. The particle size distribution of the nanoemulsions were determined using DLS. The In vitro release profile of paclitaxel was obtained by incubating $20 \mathrm{~mL}$ of the nanoemulsions $\left(0.3 \mathrm{mg} \mathrm{mL} \mathrm{m}^{-1}\right.$ of paclitaxel) in a Spectra/Por dialysis bag $(\mathrm{MWCO}=12 \mathrm{kDa})$ placed in $100 \mathrm{~mL}$ of PBS containing various percentages of methanol. At pre-determined time intervals, a $1 \mathrm{~mL}$ aliquot of the medium was taken, freeze-dried, and dissolved in acetonitrile to determine the concentration of paclitaxel using reversed phase high performance liquid chromatography (RP-HPLC).

\section{Micro-CT analysis}

HeLa cells were seeded onto a $100 \mathrm{~mm}$ dish at a density of $10^{7}$ cells per dish, followed by incubation in DMEM at $37{ }^{\circ} \mathrm{C}$ for $24 \mathrm{~h}$. The medium was then replaced by $10 \mathrm{~mL}$ of fresh DMEM containing the nanoemulsions, followed by incubation for $4 \mathrm{~h}$. The cells were trypsinized and collected by centrifugation, and then fixed using $4 \%$ formaldehyde at $4{ }^{\circ} \mathrm{C}$ overnight. The cells were then mixed with $1 \%$ agarose gel in a $1.5 \mathrm{~mL}$ tube and visualized on a micro-CT (Simens Inveon Micro-CT, Siemens Medical Solutions, Knoxville, TN, USA).

\section{Cellular uptake of fluorescently labeled Nanoemulsions}

To probe the cellular uptake of lipiodol nanoemulsions, 1.64 wt-\% of Nile red was incorporated in the core of the nanoemulsions. HeLa cells were seeded in a chamber slide (4-well Culture Slides, BD Falcon, MA) at a density of $10^{5}$ cells per well, and pre-incubated in DMEM at $37{ }^{\circ} \mathrm{C}$ for $24 \mathrm{~h}$. The medium was then replaced by Nile red-loaded nanoemulsions at a concentration of $1.2 \mu \mathrm{g}$ $\mathrm{mL}^{-1}$ in a fresh medium, and the cells were treated for $4 \mathrm{~h}$ at $37{ }^{\circ} \mathrm{C}$. Afterwards, the nanoemulsions were removed, and the cells were washed several times with PBS. Then the cells were fixed using $4 \mathrm{wt}$ \% formaldehyde for $30 \mathrm{~min}$ at room temperature. The nuclei of cells were stained using DAPI $\left(1.5 \mathrm{mg} \mathrm{mL}^{-1}\right)$ for $2 \mathrm{~min}$, and 
the specimens were examined using a confocal laser scanning microscope (LSM510, Carl Zeiss, Oberkochen, Germany). Confocal images were quantitatively analyzed using ImageJ (U. S. National Institutes of Health, Bethesda, Maryland, USA).

\section{Cytotoxicity}

HeLa cells were cultured in DMEM (10\% FBS and 1\% penicillin/streptomycin) at $37{ }^{\circ} \mathrm{C}$. The cells were seeded onto a 96-well-plate at a density of $10^{4}$ cells per well, followed by incubation at $37^{\circ} \mathrm{C}$ for $24 \mathrm{~h}$. The medium was replaced by a fresh DMEM containing pNEs at various concentrations. The cells were then incubated for $4 \mathrm{~h}$, and the pNEs were removed from the culture medium. The cells were further incubated for 2 days in a fresh medium, and then treated with a CCK- 8 assay solution. The absorbance at $450 \mathrm{~nm}$ was recorded using a microplate reader (CLARIO Star, BMG Labtech, Germany).

\section{Results}

The synthetic scheme of PG- $b$-PCL with a linear block of PG is summarized in Fig. 1a. The hydroxyl group of glycidol was temporarily protected through the reaction of glycidol with ethyl vinyl ether. The resulting EEGE was then polymerized by ring-opening polymerization to PEEGE. The synthesized PEEGE was used as an initiator for the ring-opening polymerization of $\varepsilon$-caprolactone to PEEGE- $b$-PCL. The hydroxyl groups of PEEGE- $b$-PCL were finally de-protected to obtain linear PG- $b$-PCL. The chemical structure of the synthesized block copolymer was analyzed using ${ }^{1} \mathrm{H}$ NMR (Fig. 1b). Peaks number ' 1 ' to ' 5 ' in the spectrum were assigned to the hydrogen atoms in PCL, and peaks number ' 6 ' to ' 8 ' to PG. The integrations of the corresponding peaks in the NMR spectrum were analyzed to calculate the molar ratio of the two blocks, as shown in Table 1. Three compositions of PG- $b$-PCL with different molar ratios of the two blocks were selected for further investigation: the molar ratios of PCL to PG were 2, 2.5, and 4.5, and the corresponding polymers are denoted as $\mathrm{PG}_{40}-b-\mathrm{PCL}_{80}$, $\mathrm{PG}_{47}-b-\mathrm{PCL}_{118}$, and $\mathrm{PG}_{32}-b-\mathrm{PCL}_{144}$ (Additional file 1: Figure S1). The subscripts represent the number of repeating units calculated from the molecular weight and the molar ratios. The number average molecular weights
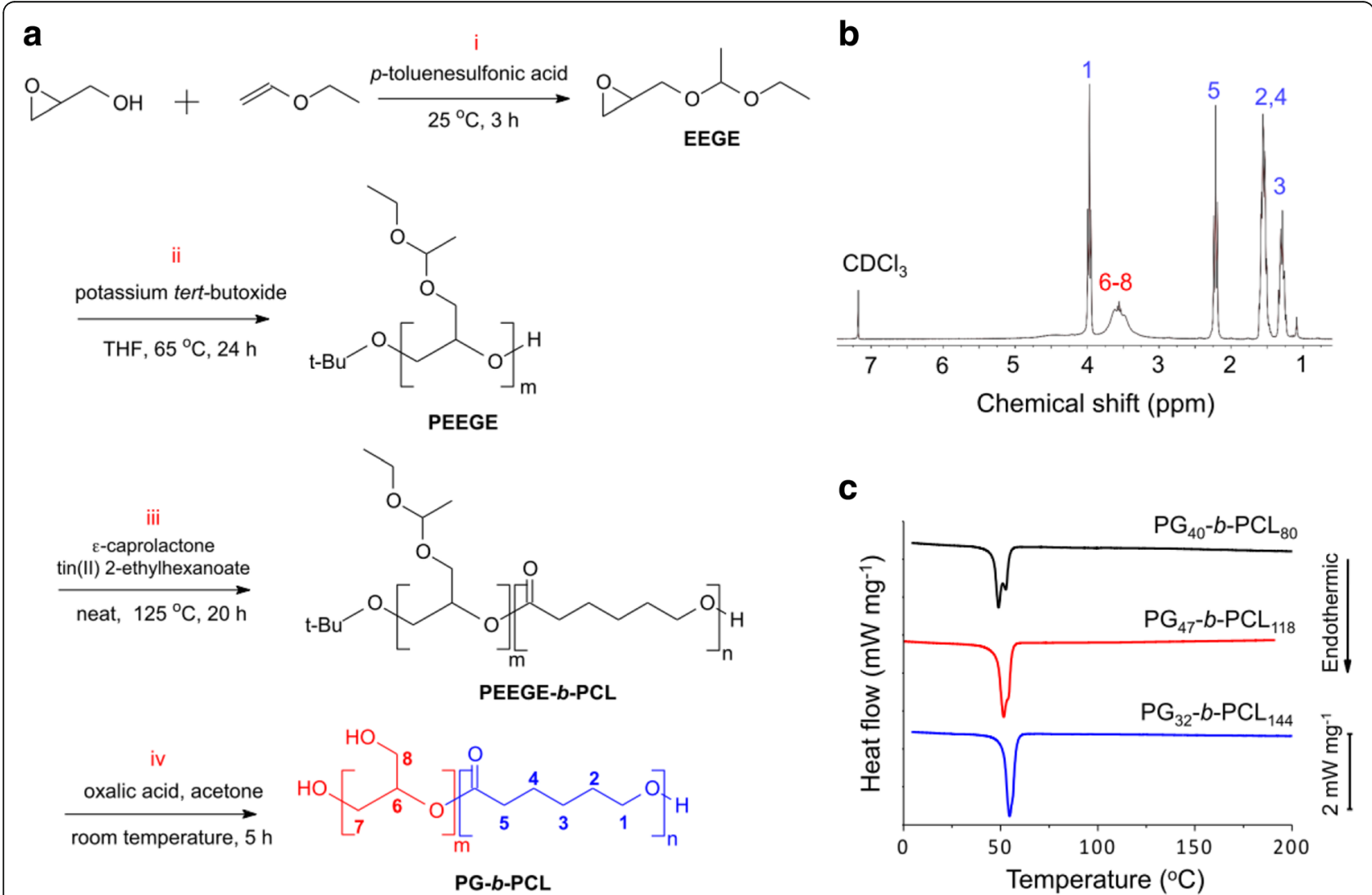

Fig. 1 Synthesis and characterization of PG-b-PCL: a Synthetic scheme of PG-b-PCL: (i) Synthesis of EEGE, (ii) Polymerization of PEEGE, (iii) Ring-opening polymerization of $\varepsilon$-caprolactone to PEEGE- $b$-PCL, and (iv) De-protection of PEEGE to synthesize PG- $b$-PCL; $\boldsymbol{b}^{1}{ }^{1} H$ NMR spectrum of $\mathrm{PG}_{40}-b-P C L_{80}$ with the corresponding functional peaks shown in the chemical structure of PG-b-PCL in (a); $\mathbf{c}$ DSC diagram of PG-b-PCL in the second heating scan 
Table 1 Molecular weights of PG-b-PCL with different lengths of $P G$ and $P C L$

\begin{tabular}{lllll}
\hline Polymer & $M_{n}^{a}$ & $M_{w}^{a}$ & $P^{a} I^{b}$ & Molar ratio of $P G: P C L^{c}$ \\
\hline $\mathrm{PG}_{40}-b-P C L_{80}$ & 6246 & 12,204 & 1.95 & $1: 2$ \\
$P_{4}-b-P C L_{118}$ & 11,250 & 16,989 & 1.51 & $1: 2.5$ \\
$P_{32}-b-P C L_{144}$ & 12,258 & 18,822 & 1.53 & $1: 4.5$ \\
\hline
\end{tabular}

${ }^{\mathrm{a}}$ Determined by GPC

${ }^{b}$ Polydispersity index $=M_{w} / M_{n}$

'Determined using ${ }^{1} \mathrm{H}$ NMR

$\left(\mathrm{M}_{\mathrm{n}}\right)$ were in the range of $6-12 \mathrm{kDa}$, and the weight average molecular weights $\left(\mathrm{M}_{\mathrm{w}}\right)$ were in the range of $12-19 \mathrm{kDa}$. The polydispersity indexes were from 1.5 to 1.9. The DSC diagrams of the three copolymers are shown in Fig. 1c. The melting temperatures $(49,52$, and $55{ }^{\circ} \mathrm{C}$ ) and crystallinity (enthalpy $=58,71$, and $67 \mathrm{~J} \mathrm{~g}^{-1}$ ) of the copolymers increased as the length of the PCL increased, which might have been caused by the crystallization of the PCL chain.

Polymeric micelles (denoted as 'bMCs') were prepared using PG- $b$-PCL with different lengths of the PCL block, and their critical aggregate concentrations (c.a.c.) were determined using pyrene. As expected, the c.a.c. value of
$\mathrm{PG}_{40}-b-\mathrm{PCL}_{80}\left(7.4 \times 10^{-3} \mathrm{~g} \mathrm{~L}^{-1}\right)$ was much higher than that of $\mathrm{PG}_{47}-b-\mathrm{PCL}_{118}$ and $\left(4.0 \times 10^{-3} \mathrm{~g} \mathrm{~L}^{-1}\right)$, and $\mathrm{PG}_{32^{-}}$ $b$ - $\mathrm{PCL}_{144}\left(3.5 \times 10^{-3} \mathrm{~g} \mathrm{~L}^{-1}\right)$ (Fig. 2a). This is because the relatively larger segment of PCL decreased c.a.c. due to its increased hydrophobicity. However, the size distribution of bMCs of $\mathrm{PG}_{32}-b-\mathrm{PCL}_{144}$ and $\mathrm{PG}_{40}-b-\mathrm{PCL}_{80}$ was significantly broader than that of $\mathrm{PG}_{47}-b-\mathrm{PCL}_{118}$ (the hydrodynamic diameter $=113.9 \mathrm{~nm}, \quad$ PDI $=0.11$ ) (Fig. 2b). The bMCs exhibit a relatively uniform spherical morphology with a diameter of $83 \pm 29 \mathrm{~nm}$ (Fig. 2c). The smaller particle size from TEM might be due to the shrinkage during the drying process. Figure $2 \mathrm{~d}$ and $\mathrm{e}$ show the size distributions and TEM image of blank lipiodol nanoemulsions (bNEs). The result indicates that bNE-1, which was prepared using $10 \mathrm{mg} \mathrm{PG}_{47}-b-\mathrm{PCL}_{118}$ and $50 \mathrm{mg}$ of lipiodol by a stirring method, had the smallest diameter $(181 \mathrm{~nm})$. Considering the physiochemical characteristics of self-assembled aggregates, we chose $\mathrm{PG}_{47}-b-\mathrm{PCL}_{118}$ for further investigation on the preparation of nanoemulsions incorporating lipiodol as a core using various emulsification parameters listed in Table 2. The loading yield and efficiency of lipiodol were 75.5 and $90.6 \%$, respectively, as determined using the

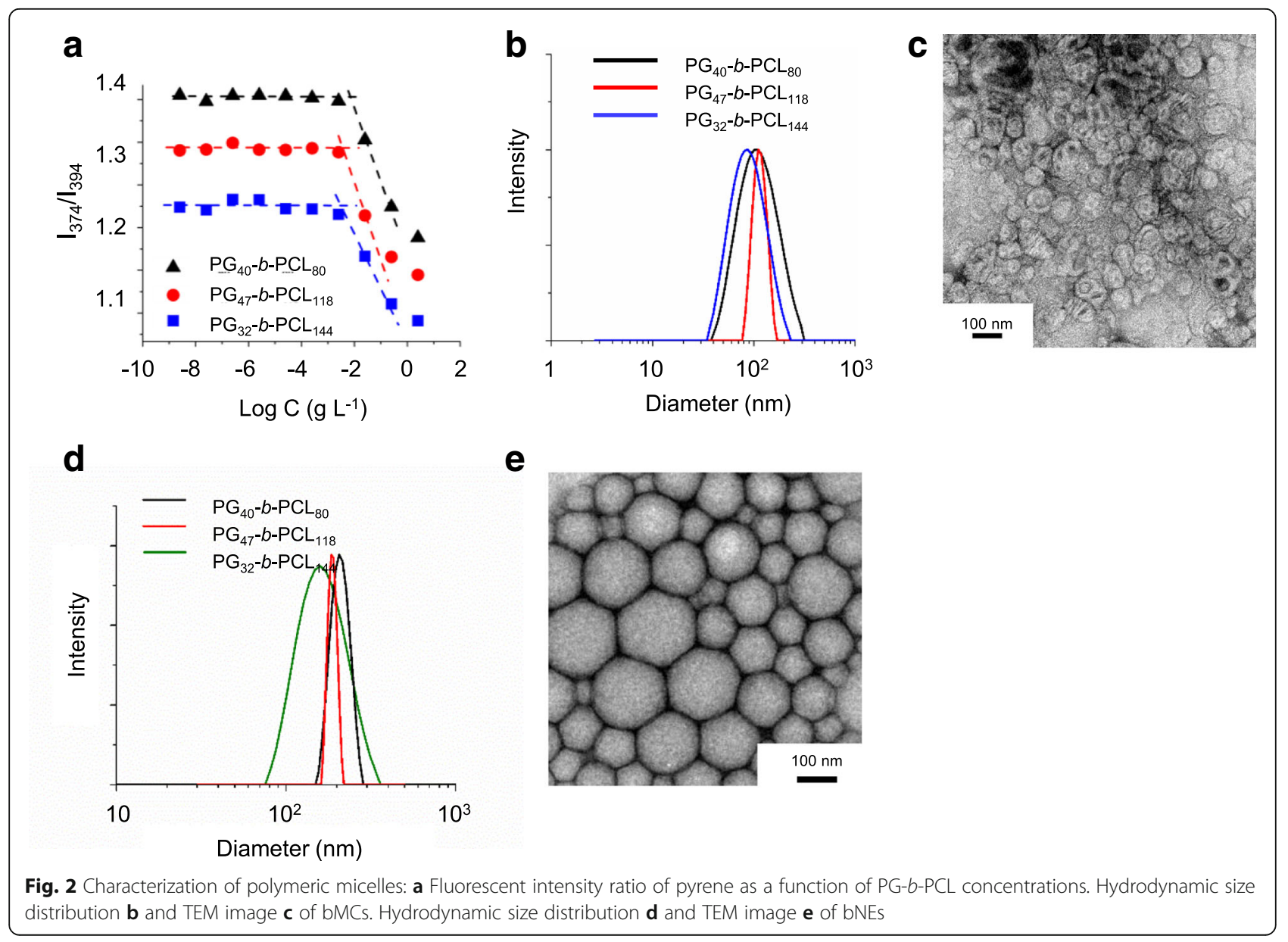


Table 2 Experimental parameters for the preparation of bNEs, mean hydrodynamic diameter, and polydispersity index

\begin{tabular}{|c|c|c|c|c|c|c|c|c|}
\hline \multirow{2}{*}{$\begin{array}{l}\text { Batch } \\
\text { No. }\end{array}$} & \multirow{2}{*}{$\begin{array}{l}m_{1} \\
(m g)^{a}\end{array}$} & \multirow{2}{*}{$\begin{array}{l}\mathrm{m}_{2} \\
(\mathrm{mg})^{\mathrm{b}}\end{array}$} & \multirow{2}{*}{$\begin{array}{l}V_{\text {acetone }} \\
(\mathrm{mL})\end{array}$} & \multicolumn{3}{|c|}{ Emulsification condition } & \multirow{2}{*}{$\begin{array}{l}\text { Mean Diameter } \\
(\mathrm{nm})^{\mathrm{c}}\end{array}$} & \multirow[t]{2}{*}{$\mathrm{PDI}^{\mathrm{d}}$} \\
\hline & & & & Stirring speed (rpm) & Temperature $\left({ }^{\circ} \mathrm{C}\right)$ & Method & & \\
\hline bNE-1 & 10 & 50 & 3 & 1000 & 45 & Magnetic stirring & 181.0 & 0.128 \\
\hline bNE-2 & 10 & 100 & 3 & 1000 & 45 & Magnetic stirring & 215.0 & 0.081 \\
\hline bNE-3 & 10 & 150 & 3 & 1000 & 45 & Magnetic stirring & 231.8 & 0.101 \\
\hline bNE-4 & 10 & 100 & 1 & $500-700$ & 25 & Magnetic stirring & 341.1 & 0.190 \\
\hline bNE-5 & 10 & 100 & 1 & $500-700$ & 45 & Magnetic stirring & 233.2 & 0.085 \\
\hline bNE-6 & 10 & 100 & 1 & 1000 & 25 & Magnetic stirring & 219.3 & 0.087 \\
\hline bNE-7 & 10 & 100 & 3 & $500-700$ & 25 & Magnetic stirring & 226.9 & 0.119 \\
\hline bNE-8 & 10 & 100 & 3 & 10,000 & 25 & Homogenization & 286.2 & 0.139 \\
\hline bNE-9 & 10 & 100 & 3 & 20,000 & 25 & Homogenization & 349.0 & 0.171 \\
\hline bNE-10 & 10 & 100 & 3 & 30,000 & 25 & Homogenization & 264.4 & 0.194 \\
\hline bNE-11 & 10 & 100 & 3 & 10,000 & 45 & Homogenization & 250.1 & 0.096 \\
\hline
\end{tabular}

${ }^{a} \mathrm{~m} 1=$ the mass of PG- $b-\mathrm{PCL}$

${ }^{\mathrm{b}} \mathrm{m} 2$ = the mass of lipiodol

'Determined by DLS

dPolydispersity index $=\mu_{2} / \Gamma^{2}$, estimated by the cumulant method

calibration curve from the absorption at $260 \mathrm{~nm}$ (Additional file 1: Figure S2).

Nanoemulsions with various concentrations of paclitaxel were prepared using $\mathrm{PG}_{47}-b-\mathrm{PCL}_{118}$, as described in Table 3, and the loading yield and efficiency were determined. The nanoemulsions fed with $3 \mathrm{mg}(2.6 \%)$ of paclitaxel (pNE-3) showed the highest encapsulation efficiency (55\%) (Fig. 3a). The lower efficiency at a higher loading yield might be caused by the limited solubility of paclitaxel in lipiodol, which is about $10 \mathrm{mg} \mathrm{mL}^{-1}$. Despite the different loading yield of paclitaxel, no significant difference in the size distribution of pNEs was observed (Fig. 3b). To evaluate the PG- $b$-PCL nanoemulsions as drug delivery vehicles, we examined the dispersion stability of pNE-3 in 10 and 50\% FBS. No significant change of their hydrodynamic diameter was observed for a week at $37^{\circ} \mathrm{C}$ (Fig. 3c). This is attributed to excellent biocompatibility and the high resistance of PG to non-specific protein adsorption.

Next, we determined the in vitro release profiles of paclitaxel from pNEs (pNE-3). Due to the negligible solubility of paclitaxel in an aqueous solution, surfactants or polar organic solvents are commonly added to the release medium to maintain the sinking condition.
However, there is no standardized experimental condition presumably because of the differences in the properties of materials used for paclitaxel formulations. In this work, a mixture of PBS and methanol was used to examine how solvent polarity affects the release of paclitaxel from pNEs (Fig. 3d). The sink condition was maintained if the volume percentage of methanol was higher than $20 \%$. In $50 \%$ methanol, $37.4 \%$ of paclitaxel was released from pNEs for $24 \mathrm{~h}$ at the initial stage. After $24 \mathrm{~h}$, the release rate became lower, and for 15 days only $54.3 \%$ of the paclitaxel was released. The drug release is controlled by slow diffusion of paclitaxel as a result of partitioning from the hydrophobic core of the nanoemulsions into 40 and $50 \%$ methanol during the first day, followed by slow release. However, in $20 \%$ methanol, the drug release was very limited to less than $20 \%$ for 2 weeks, and no significant release of pactlitaxel was observed in $10 \%$ methanol. The results indicate that the hindered diffusion of paclitaxel through the PCL-based interphase is a limiting factor for the diffusion of paclitaxel from the lipiodol core to the release medium.

To achieve active targeting via FA receptor (FAR)-mediated endocytosis, pNEs were conjugated with FA by boronic acid ester linkage to produce fpNEs as described

Table 3 Experimental parameters for pNE, mean hydrodynamic diameter, and polydispersity index

\begin{tabular}{|c|c|c|c|c|c|c|}
\hline Batch No. & Weight $\mathrm{PG}_{47}-b-\mathrm{PCL}_{118}(\mathrm{mg})$ & mLipiodol $(\mathrm{mg})$ & mpaclitaxel (mg) & $V_{\text {acetone }}(\mathrm{ml})$ & Mean Diameter ${ }^{\mathrm{a}}(\mathrm{nm})$ & $\mathrm{PDI}^{\mathrm{b}}$ \\
\hline pNE-1 & 12.3 & 59.8 & 1.2 & 3 & 174.0 & 0.090 \\
\hline pNE-2 & 12.0 & 56.4 & 2.1 & 3 & 166.2 & 0.063 \\
\hline pNE-3 & 12.3 & 56.5 & 3.3 & 3 & 158.9 & 0.065 \\
\hline pNE-4 & 10.6 & 51.4 & 5.1 & 3 & 171.1 & 0.094 \\
\hline
\end{tabular}

${ }^{\mathrm{a}}$ Determined by DLS

bolydispersity index (PDI) $=\mu_{2} / \Gamma^{2}$, estimated by the cumulant method

Emulsification condition of every batch is the same with that of bNE-1 (magnetic stirring at $10,000 \mathrm{rpm}$ at $40-50^{\circ} \mathrm{C}$ ) 

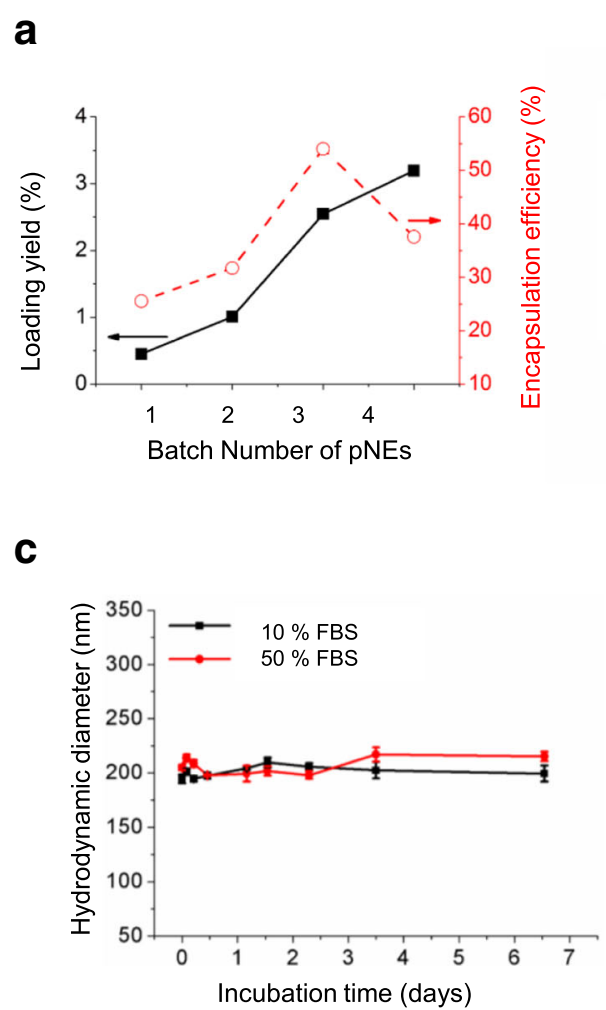
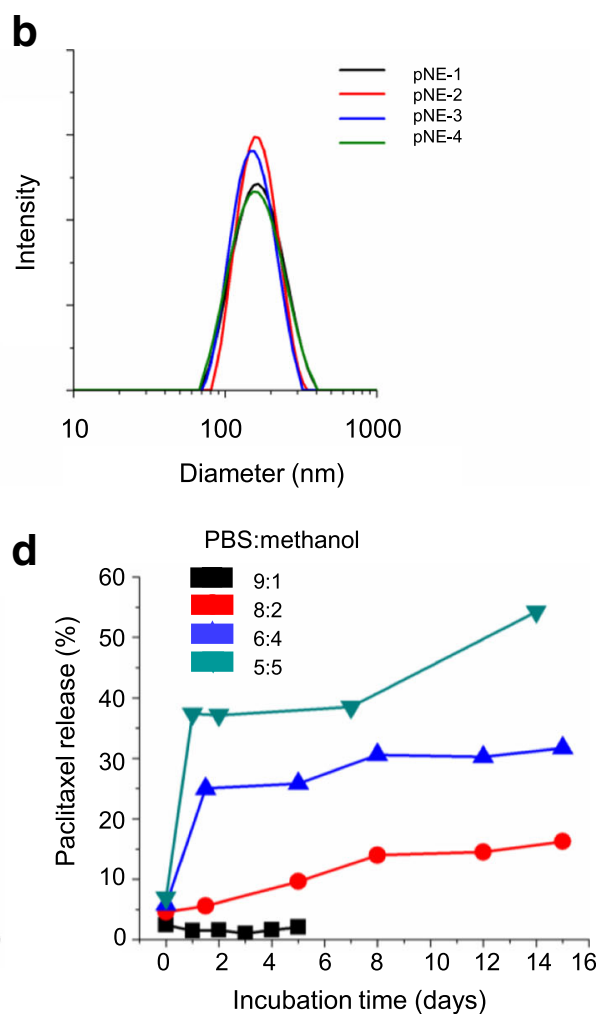

Fig. 3 Preparation and characterization of paclitaxel loaded nanoemulsion (pNE): a Loading yield and encapsulation efficiency of paclitaxel within lipiodol nanoemulsions. b Size distribution of pNEs. c Dispersion stability of pNEs in PBS containing 10 and $50 \%$ FBS at $37{ }^{\circ} \mathrm{C}$. $\mathbf{d}$ In vitro release profiles of paclitaxel from pNEs in a mixture of PBS and methanol

in the experimental section. Boronic acid binds to alcohol with high affinity through the formation of boronate ester, which is a strong single-pair reversible functional group, interaction in an aqueous milieu [10]. FBA was synthesized through simple amide coupling between FA and 3-aminophenylboronic acid. Boronic acid can simply react with the vicinal diol groups at the end of the PG block to form double ester linkages with each boronate unit with a degree of folate substitution (19.2\%).

To visualize the cellular uptake of pNEs, Nile red was incorporated as a hydrophobic fluorescent dye into the nanoemulsions. HeLa cells, which overexpress FAR type $\alpha$, were incubated with the nanoemulsions loaded with 1.64 wt- $\%$ Nile red, and examined using a confocal fluorescence microscope (Fig. 4a). The Nile red-loaded nanoemulsions are shown in red, while DAPI staining showed the area and distribution of the nuclei. The fluorescence intensity per individual cell is represented in Fig. 4b. The average intensity per single cell was almost twice higher in the cells treated with fpNEs than those treated with pNEs. These results indicated that the FA conjugation efficiently increased the cellular uptake of the nanoemulsions presumably through binding to FARs in the cellular membrane. Moreover, assuming that the density of PG- $b$-PCL is $1.1 \mathrm{~g} \mathrm{~mL}^{-1}$, FA ligands were located on the surface of nanoemulsions at a density of $6.8 \mathrm{FA}$ molecules per $\mathrm{nm}^{2}$ [31]. It can be inferred that the high density of FA on the nanoemulsions enhanced the cellular uptake efficiency by multivalent binding to HeLa cells. It was also confirmed by competition assay of folate receptor mediated intracellular uptake of fpNEs. In case that folate receptors are blocked by cultivating cells in folic acid-rich medium, the intracellular uptake efficiency of fpNEs decreased to $30.7 \%$ of folate receptor open system (Additional file 1: Figure S3).

Lipiodol within the nanoemulsions can serve as a CT contrast agent, so the diagnostic capability of the lipiodol nanoemulsions was evaluated using micro-CT analysis. HeLa cells were treated with PBS, pNEs, and fpNEs, followed by the fixation in an agarose gel phantom (Fig. 4c). The emulsions concentration was varied to achieve from 10 to $100 \mu \mathrm{g} \mathrm{mL} \mathrm{m}^{-1}$ of lipiodol. The CT values of the cells $\left(1 \times 10^{7}\right)$ treated with fpNEs and pNEs, where the concentration of lipoidol was $10 \mu \mathrm{g} \mathrm{mL} \mathrm{m}^{-1}$, were $73.4 \pm 3.6$ and $40.3 \pm 1.4 \mathrm{HU}$, respectively, where $\mathrm{HU}$ is a hounsfield unit (Fig. 4d). The CT value increased with increasing the concentration of lipiodol. The CT values of cells treated with pNEs were $82.0 \pm 0.9$ and $126.4 \pm 3.8$ $\mathrm{HU}$ at 50 and $100 \mu \mathrm{g} \mathrm{mL} \mathrm{m}^{-1}$ of lipiodol, respectively. The values of cells treated with fpNEs were $148.7 \pm 3.1$ and 

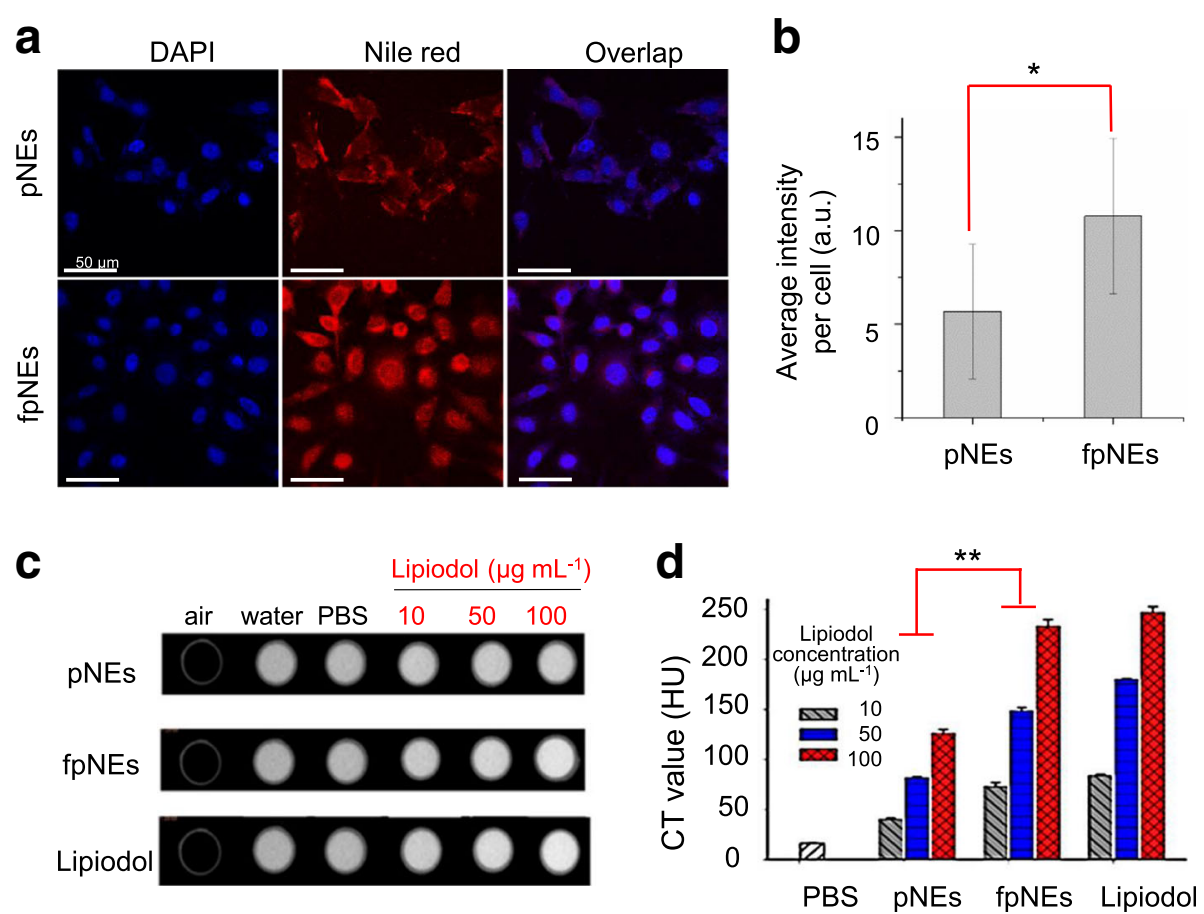

Fig. 4 Enhanced intracellular uptake of folate deocorated nanoemulsions (fNEs): a Confocal fluorescence microscopic images of HeLa cells incubated with Nile red-labeled nanoemulsions with and without FA conjugation. Scale bars $=50 \mu \mathrm{m}$. $\mathbf{b}$ The average intensity of individual cells ( $\left.{ }^{*} p=0.001\right)$. Micro-CT images $\mathbf{c}$ and $\mathrm{HU}$ values $\mathbf{d}$ of phantoms of HeLa cells treated with deionized water, PBS, pg-pNE, fpg-pNE, and lipiodol $\left(n=3\right.$, Error bars indicate standard deviation, $\left.{ }^{* *} p<0.05\right)$

$233.4 \pm 6.4 \mathrm{HU}$ at 50 and $100 \mu \mathrm{g} \mathrm{mL} \mathrm{L}^{-1}$ of lipiodol, respectively. These values were much higher than those from treatment with pNEs. The CT values of most soft tissues are in the range of 30-100 $\mathrm{HU}$, and the difference of 50$100 \mathrm{HU}$ can be used to distinguish the tissues of interest. Therefore, it can be said that fpNEs are applicable as contrast agents for CT imaging of tumors. Interestingly, regardless of the lipiodol concentration, the $\mathrm{CT}$ value of fpNEs was about 1.8 times higher than that of pNEs, which corresponds to the difference in the fluorescence intensity per cell. The difference seems to result from the cellular uptake efficiency of nanoemulsions. Compared with our previous study on the feasibility of CT imaging using lipiodol nanoemulsions [30] (therein, denoted 's $r$-NEs') composed of LPEI- $g$-cholesterol, cholesterol, DSPE-PEG, siRNAs, and paclitaxel, the contrast enhancement by fpNEs was 1.8 -fold better than that from the use of $s r$-NEs. This was true even with the lesser amount of lipiodol (ca. $72 \%$ of fpNEs), probably due to difference in the cellular uptake. Thus, it is expected that fpNEs could be further developed as an in vivo tumor-targeting $\mathrm{CT}$ contrast agent.

The effects of FA conjugation on the cytotoxicity of paclitaxel-loaded lipiodol nanoemulsions were examined using HeLa cells (Fig. 5). bMCs and bNEs were also used as negative controls to determine the cytotoxicity of
$\mathrm{PG}_{47}-b-\mathrm{PCL}_{118}$ and lipiodol. The bMCs and bNEs exhibited no significant cytotoxicity up to a micelle concentration of $1.2 \mu \mathrm{g} \mathrm{mL} \mathrm{m}^{-1}$. In contrast, both pNEs and fpNEs caused efficient death of target cells. At $1.2 \mu \mathrm{g} \mathrm{mL} \mathrm{m}^{-1}$ of nanoemulsions, pNEs showed about $53 \%$ cell viability on HeLa cells at a paclitaxel concentration of $30 \mathrm{ng} \mathrm{mL}$,

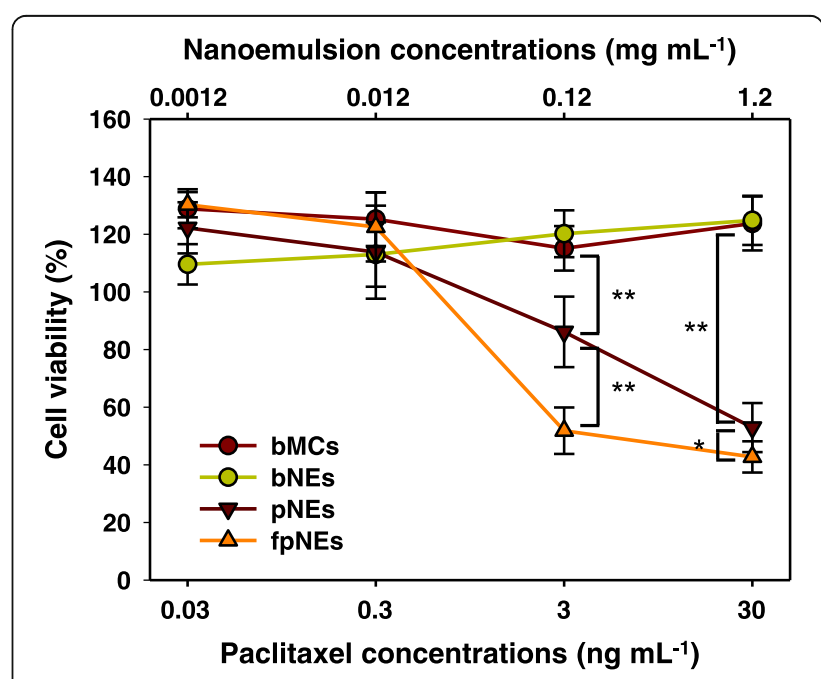

Fig. 5 Cytotoxicity to HeLa cells of nanoemulsions with and without FA conjugation with different concentrations of paclitaxel. bMCs and bNEs were used as negative controls $\left({ }^{*} p<0.1,{ }^{* *} p<0.01\right)$ 
while fpNEs showed $41 \%$ cell viability at the same concentration. However, at $3 \mathrm{ng} \mathrm{mL}^{-1}$, fpNEs exhibited $52 \%$ cell viability while pNEs had $85 \%$. The result indicates that the FA conjugation to the surface of lipiodol nanoemulsions effectively promotes the intracellular delivery of the nanocarriers to boost the efficacy of paclitaxel.

\section{Discussion}

Although most widely used as a hydrophilic corona of polymeric nanoparticles for biomedical applications, PEG has several limitations such as lowering cellular uptake of nanoparticles, a lack of responsiveness to environmental changes, and insufficient number of sites for chemical modification. Hence, a development of a novel hydrophilic moiety is highly demanded. PG is one of the most prominent alternatives to PEG due to tis excellent dispersion stability and biocompatibility. We synthesized PG- $b$-PCL amphiphilic diblock copolymers having critical aggregation concentrations in the range of $10^{-6}-10^{-7} \mathrm{M}$ which is comparable to that of PEG based polymeric emulsifiers. Theranostic nanoemulsions co-encapsulating lipiodol and paclitaxel were prepared using $\mathrm{PG}_{47}-b-\mathrm{PCL}_{118}$.

The potency of our theranostic nanoemulsions is attributed to the high loading yield of paclitaxel in lipiodol core and the high degree of cell-targeting ligand on the PG corona. As reported previously, to achieve efficient drug encapsulation, it is critical to select a suitable surrounding medium which has a high miscibility and solubility to the payloads. Besides serving as a CT contrast agent, lipiodol which has paclitaxel solubility of $10 \mathrm{mg}$ $\mathrm{mL}^{-1}$ was a good medium for encapsulated paclitaxel. Moreover, lipiodol played a role for a reservoir of paclitaxel by hindering diffusion of paclitaxel from the core through the PCL-based interphase. Considering that small molecular payloads of nanoparticles are easily diffused out during blood circulation and found in plasma or accumulated in non-target-tissues like kidney, this encapsulation stability resulted from lipiodol core can be one of the outstanding advantage for the delivery efficiency in vivo.

In addition, multiple hydroxyl groups on the PG's backbone enable to introduce a high degree of hydrophobic cancer-targeting ligand on the surface of nanoemulsions without a loss of dispersion stability in aqueous. The conjugation strategy between the vicinal diol of PG and the folate boronic acid (FBA) addressed the laborious work for introducing targeting moiety to nanoemulsions and simplified the process by enabling post-modification of nanoemulsion surface in aqueous media. The folate decorated nanoemulsions increased intracellular uptake by HeLa cells via receptor-mediated endocytosis, which consequently led to enhancement of CT imaging and anticancer therapeutic effect.
Although the theranostic efficacy of PG-PCL lipiodol nanoemulsion was only evaluated by in vitro assay in this study, it is expected that this nanoemulsion system could be further developed as an in vivo tumor-targeting theranostic contrast agent.

\section{Conclusion}

This study demonstrated that PG-b-PCL diblock copolymers can serve as a semi-solid polymeric emulsifier for theranostic nanoemulsions for cancer. The PCL block can generate a robust polymeric layer at the oil/water interface because it is not soluble in lipiodol. The PG block has a hydroxyl group in each monomeric unit, exhibiting excellent biocompatibility and anti-fouling property. In vitro cell studies with HeLa cells demonstrated that ligand conjugation efficiently increased the cellular uptake of PG- $b$-PCL nanoemulsions. The FA-conjugated lipiodol nanoemulsions loaded with $2.6 \%$ paclitaxel showed excellent anti-cancer activity $\left(\mathrm{LD}_{50}<3 \mathrm{ng} \mathrm{mL}^{-1}\right.$ ), while placebo nanoemulsions did not show any significant cytotoxicity at the same concentration. In addition, the lipiodol nanoemulsions greatly enhanced the CT contrast of tissue-mimicking phantoms of HeLa cells due to efficient intracellular translocation. This study suggests that PG- $b$-PCL can serve as a robust polymeric emulsifier to stabilize nanoemulsions while enabling facile surface functionalization with multiple targeting molecules for targeted delivery of therapeutic and imaging agents.

\section{Additional file}

Additional file 1: Supporting information. ${ }^{1} \mathrm{H}$ NMR of $\mathrm{PG}-b-\mathrm{PCL}, \mathrm{UV}-\mathrm{V}$ is absorption spectra of lipiodol nanoemulsions, and confocal fluorescence microscopic images of HeLa cells. (DOCX $960 \mathrm{~kb}$ )

\section{Abbreviations}

bMCs: Placebo polymeric micelles; bNE: Placebo nanoemulsions;

CT: Computer tomography; EEGE: Ethoxyethyl glycerol ether; FA: Folic acid; FBA: Folate boronic acid; fpNE: Folate-decorated paclitaxel-loaded nanoemulsion; PCL: Poly( $\varepsilon$-caprolactone); PEEGE: Poly(ethoxyethyl glycerol ether); PEG: Polyethylene glycol; PG: Polyglycerol; PG-b-PCL: Linear polyglycerolpoly( $\varepsilon$-caparolactone) diblock copolymer

\section{Acknowledgements}

Not applicable

\section{Funding}

This study was supported by a grant of the Korea Healthcare Technology R\&D Project, Ministry of Health \& Welfare, Republic of Korea (Grant No.: HN12C0064).

Availability of data and materials

The data sets and materials supporting the results of this article are included in the article.

\section{Authors' contributions}

YSN and JSK conceived the project and designed the experiments. THL performed the experiments and analysed the data. JHK performed micro-CT imaging. HJ synthesized and analysed FA conjugation. KP reviewed micro-CT imaging. THL, JSK, and YSN co-wrote the manuscript. All authors read and approved the manuscript. 


\section{Ethics approval}

Manuscript does not include human ethics value. Hence no consent is needed.

\section{Consent for publication}

The manuscript has been submitted with the consent of all authors and data of any other person not included.

\section{Competing interests}

The authors declare that they have no competing interests.

\section{Publisher's Note}

Springer Nature remains neutral with regard to jurisdictional claims in published maps and institutional affiliations.

\begin{abstract}
Author details
'Department of Materials Science and Engineering, Korea Advanced Institute of Science and Technology, 291 Daehak-ro, Yuseong-gu, Daejeon 34141, Republic of Korea. ${ }^{2}$ Samsung Medical Center, Samsung Biomedical Research Institute, Irwon-dong, Gangnam-gu, Seoul 06351, Republic of Korea. ${ }^{3}$ Samsung Biomedical Research Institute and Division of Hematology and Oncology, Department of Medicine, Samsung Medical Center, Sungkyunkwan University School of Medicine, Irwon-dong, Gangnam-gu, Seoul 06351, Republic of Korea. ${ }^{4}$ Department of Materials Science and Engineering and KAIST Institute for the NanoCentury, Korea Advanced Institute of Science and Technology, 291 Daehak-ro, Yuseong-gu, Daejeon 34141, Republic of Korea.
\end{abstract}

Received: 17 July 2017 Accepted: 11 October 2017

Published online: 17 October 2017

\section{References}

1. Torchilin VP. Multifunctional nanocarriers. Adv Drug Deliv Rev. 2012;64:302-15.

2. Husseini GA, Pitt WG. Micelles and nanoparticles for ultrasonic drug and gene delivery. Adv Drug Deliv Rev. 2008;60:1137-52.

3. Pasut $G$, Veronese FM. Polymer-drug conjugation, recent achievements and general strategies. Prog Polym Sci. 2007:32:933-61.

4. Roberts MJ, Bentley MD, Harris JM. Chemistry for peptide and protein PEGylation. Adv Drug Deliv Rev. 2012;64:116-27.

5. Romero-Vargas Castrillón S, Lu X, Shaffer DL, Elimelech M. Amine enrichment and poly(ethylene glycol) (PEG) surface modification of thin-film composite forward osmosis membranes for organic fouling control. J Membr Sci. 2014:450:331-9.

6. Stanzione F, Jayaraman A. Computational design of oligopeptide containing poly(ethylene glycol) brushes for stimuli-responsive drug delivery. J Phys Chem B. 2015:119:13309-20.

7. Krishnan S, Weinman CJ, Ober CK. Advances in polymers for anti-biofouling surfaces. J Mater Chem. 2008;18:3405-13.

8. Ohguchi Y, Kawano K, Hattori Y, Maitani Y. Selective delivery of folate-PEG linked, nanoemulsion-loaded aclacinomycin a to KB nasopharyngeal cells and xenograft: effect of chain length and amount of folate-PEG linker. J Drug Target. 2008;16(9):660-7.

9. Wibowo AS, Singh M, Reeder KM, Carter JJ, Kovach AR, Meng W, et al. Structures of human folate receptors reveal biological trafficking states and diversity in folate and antifolate recognition. Proc Natl Acad Sci U S A. 2013;110:15180-8.

10. Scarano W, Duong HTT, Lu H, De Souza PL, Stenzel MH. Folate conjugation to polymeric micelles via boronic acid ester to deliver platinum drugs to ovarian cancer cell lines. Biomacromolecules. 2013;14(4):962-75.

11. Garamus VM, Maksimova TV, Kautz H, Barriau E, Frey H, Schlotterbeck U, et al. Hyperbranched polymers: structure of hyperbranched polyglycerol and amphiphilic poly(glycerol ester)s in dilute aqueous and nonaqueous solution. Macromolecules. 2004;37(22):8394-9.

12. Adeli M, Namazi H, Hönzke S, Hedtrich S, Keilitz J, Haag R. Synthesis of multiarm star copolymers based on polyglycerol cores with polylactide arms and their application as nanocarriers. RSC Adv. 2015;5:14958-66.

13. Wurm F, Klos J, Räder HJ, Frey H. Synthesis and noncovalent protein conjugation of linear-hyperbranched PEG-poly(glycerol) a, $\omega_{n}$-telechelics. J Am Chem Soc. 2009:131(23):7954-5.

14. Wurm F, Nieberle J, Frey H. Double-hydrophilic linear-hyperbranched block copolymers based on poly(ethylene oxide) and poly(glycerol). Macromolecules. 2008;41(4):1184-8.
15. Son S, Shin E, Kim BS. Redox-degradable biocompatible hyperbranched polyglycerols: synthesis, copolymerization kinetics, degradation, and biocompatibility. Macromolecules. 2015;48(3):600-9.

16. Du C, Mendelson AA, Guan Q, Chapanian R, Chafeeva I, Roza G, et al. The size-dependent efficacy and biocompatibility of hyperbranched polyglycerol in peritoneal dialysis. Biomaterials. 2014;35(5):1378-89.

17. Cai T, Li M, Neoh KG, Kang ET. Surface-functionalizable membranes of polycaprolactone-click-hyperbranched polyglycerol copolymers from combined atom transfer radical polymerization, ring-opening polymerization and click chemistry. J Mater Chem B. 2013;1(9):1304-15.

18. Adeli M, Kakanejadifard A, Khani M, Bani F, Kabiri R, Sadeghizad M. A polyglycerol-polycaprolactone-polycitric acid copolymer and its selfassembly to produce medium-responsive nanoparticles. J Mater Chem B. 2014;2(23):3589-96.

19. Giacomelli C, Schmidt V, Borsali R. Nanocontainers formed by self-assembly of poly(ethylene oxide)- $b$-poly(glycerol monomethacrylate)-drug conjugates. Macromolecules. 2007:40(6):2148-57.

20. Mugabe C, Liggins RT, Guan D, Manisali I, Chafeeva I, Brooks DE, et al. Development and in vitro characterization of paclitaxel and docetaxel loaded into hydrophobically derivatized hyperbranched polyglycerols. Int J Pharm. 2011;404(1-2):238-49.

21. Yoo HS, Kwon SM, Kim YJ, Chung H, Kwon IC, Kim J, et al. Cationic lipid emulsions containing heavy oils for the transfection of adherent cells. J Control Release. 2004;98(1):179-88.

22. Idée JM, Guiu B. Use of Lipiodol as a drug-delivery system for transcatheter arterial chemoembolization of hepatocellular carcinoma: a review. Crit Rev Oncol Hematol. 2013;88(3):530-49.

23. Xie J, Lee S, Chen X. Nanoparticle-based theranostic agents. Adv Drug Deliv Rev. 2010;62:1064-79.

24. Le Kim TH, Jun H, Kim JS, Nam YS. Stable nanoemulsions prepared via interfacial solidification of amphiphilic polyether-polyester block copolymers. J Colloid Interface Sci. 2015;443:197-205.

25. Nam YS, Kim JW, Park J, Shim J, Lee JS, Han SH. Tocopheryl acetate nanoemulsions stabilized with lipid-polymer hybrid emulsifiers for effective skin delivery. Colloids Surfaces B Biointerfaces. 2012:94:51-7.

26. Nam YS, Kim JW, Shim J, Han SH, Kim HK. Nanosized emulsions stabilized by semisolid polymer interphase. Langmuir. 2010;26(16):13038-43.

27. Nam YS, Kim JW, Shim J, Han SH, Kim HK. Silicone oil emulsions stabilized by semi-solid nanostructures entrapped at the interface. J Colloid Interface Sci. 2010;351(1):102-7

28. Yoon CJ, Chung JW, Park JH, Yoon YH, Lee JW, Jeong SY, et al. Transcatheter arterial chemoembolization with paclitaxel-lipiodol solution in rabbit VX2 liver tumor. Radiology. 2003;229(1):126-31.

29. Bae KH, Lee Y, Park TG. Oil-encapsulating PEO-PPO-PEO/PEG shell crosslinked nanocapsules for target-specific delivery of paclitaxel. Biomacromolecules. 2007:8(2):650-6.

30. Oh MH, Kim JS, Lee JY, Park TG, Nam YS. Radio-opaque theranostic nanoemulsions with synergistic anti-cancer activity of paclitaxel and BCl-2 siRNA. RSC Adv. 2013;3(34):14642-51.

31. Hong S, Leroueil PR, Majoros IJ, Orr BG, Baker JR, Banaszak Holl MM. The binding avidity of a nanoparticle-based multivalent targeted drug delivery platform. Chem Biol. 2007;14(1):107-15.

\section{Submit your next manuscript to BioMed Central and we will help you at every step:}

- We accept pre-submission inquiries

- Our selector tool helps you to find the most relevant journal

- We provide round the clock customer support

- Convenient online submission

- Thorough peer review

- Inclusion in PubMed and all major indexing services

- Maximum visibility for your research

Submit your manuscript at www.biomedcentral.com/submit
) Biomed Central 